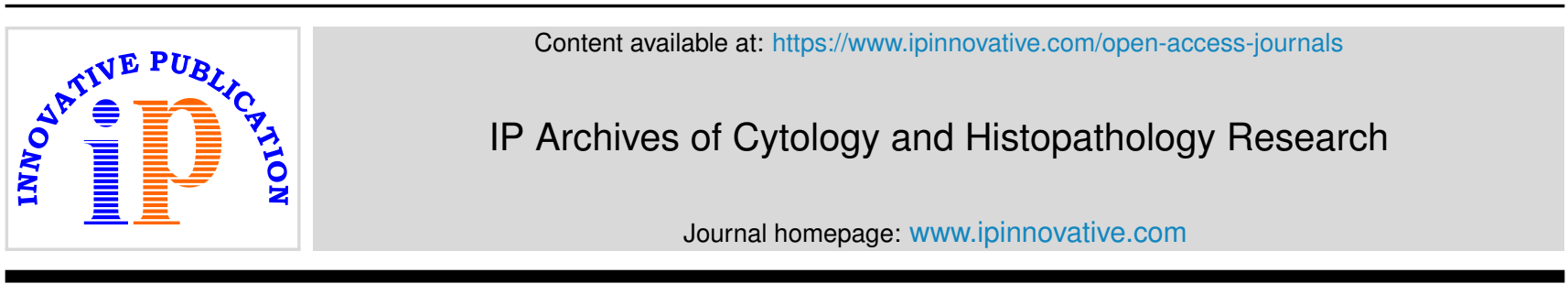

Original Research Article

\title{
A Prospective Study for comparison of diagnostic utility of Gene XPERT MTB/RIF Assay, adenosine deaminase and cytology in Tuberculous Pleural Effusion
}

\author{
Archana Garg ${ }^{1}$, Lakshmi Agarwal ${ }^{2, *}$, Mamta Gupta $^{3}$, Rishab Mathur ${ }^{4}$ \\ ${ }^{1}$ Dept. of Microbiology, Govt. Medical College, Kota, Rajasthan, India \\ ${ }^{2}$ Dept. of Pathology, Govt. Medical College, Kota, Rajasthan, India \\ ${ }^{3}$ Govt. Medical College, Baripada, Odisha, India \\ ${ }^{4}$ Govt. Medical College, Kota, Rajasthan, India
}

\section{A R T I C L E I N F O}

Article history:

Received 22-09-2020

Accepted 26-09-2020

Available online 20-10-2020

Keywords:

Tuberculosis

Xpert MTB/RIF

Adenosine deaminase

Lymphocytes

Pleural fluid

\begin{abstract}
A B S T R A C T
Introduction: Tubercular pleural effusion is the second most common extrapulmonary form of tuberculosis in India. It poses several health challenges in a developing country like India which has limited resources, for developing appropriate methods to diagnose.

Aim: The objective of the study was to determine the role of cartridge-based nucleic acid amplification test (CBNAAT) in the diagnosis of tubercular pleural effusion (TPE) and compare it with the diagnostic utility of Adenosine Deaminase (ADA) and lymphocyte counts in pleural fluid.

Methods: Total 100 patients were selected from July 2019 to December 2019. Pleural fluid was collected in sterile container. Gene/xpert, Adenosine deaminase, total leukocytes count and lymphocytes percentage were evaluated.

Results: It was found that high leukocytes count and lymphocyte predominance was present in $>80 \%$ of the patient. Mean ADA was $68.7 \mathrm{U} / \mathrm{L} \pm 13.2$ (SD). CBNAAT was positive in 30 patients. Acid fast bacilli stain was negative in the entire patient.

Conclusion: The usefulness of Xpert MTB/RIF to diagnose pleural TB is limited by its poor sensitivity. A high ADA $\geq 40 \mathrm{U} / \mathrm{L}$ in combination with Light's criteria to define exudates, with lymphocyte predominance is sufficient evidence to diagnose tuberculous pleural effusion and initiate anti-tubercular therapy, thereby deferring the need to perform an invasive pleural biopsy.

(C) 2020 Published by Innovative Publication. This is an open access article under the CC BY-NC license (https://creativecommons.org/licenses/by-nc/4.0/)
\end{abstract}

\section{Introduction}

Tuberculosis (TB) is one of the deadliest infectious diseases caused by Mycobacterium tuberculosis. It is one of the top 10 causes of death worldwide. Approximately a third of the world's populations who are infected with Mycobacterium tuberculosis are at risk of developing TB disease.

Pulmonary TB is the most common form of TB, with extrapulmonary tuberculosis (EPTB) in the form of pleural effusion, accounting for $\sim 15 \%$ of cases. It may increases to $50 \%$ in high HIV prevalence settings. ${ }^{1}$

\footnotetext{
* Corresponding author.

E-mail address: drlaxmiagarwal@gmail.com (L. Agarwal).
}

Mycobacteria infect the pleura and pleural space. This initiates the delayed hypersensitivity reaction resulting in the increase in fluid formation and decreases its removal. ${ }^{2}$ There is increase in neutrophilic infiltrate initially followed by lymphocyte driven immune reaction and granuloma formation. This further increases the release of adenosine deaminase (ADA).

Diagnosis of EPTB is a challenge due to the paucibacillary and non-uniform distribution of microorganisms and the variable clinical presentation. Clinical guidelines for diagnosis alone can lead to over-diagnosis and treatment which can increase the resistant strain and also the mortality and morbidity of the patient. 
The gold standard for diagnosis of tuberculous pleural effusion (TPE) depends on the demonstration of tubercle bacilli in pleural fluid either by culture or AFB positive and granuloma formation in pleural biopsy specimen and fluid. Each test has its own limitations.

Due to paucibacillary nature of pleural fluid, direct lung involvement may not occur, and is found to be positive in less than $5 \%$ of cases. ${ }^{3}$ Culture of pleural fluid also has low sensitivity (24-58\%) and time consuming as it takes approximate 2 to 8 weeks. ${ }^{4,5}$

Thoracoscopic pleural biopsy is an invasive, time consuming procedure and is associated with risk, with a sensitivity ranging from 93 to $100 \% .{ }^{6-9}$ Rapid identification is essential for early treatment initiation and improved patient outcome

To overcome above limitations newer methods has been developed. One among many is

GeneXpert MTB/RIF assay, a fully automated quantitative real-time hemi-nested PCR which can detect Mycobacterium tuberculosis complex directly from clinical samples and also rifampicin susceptibility in less than 2 hours. It is recently endorsed by the WHO as a rapid test for both smear-positive and smear-negative (paucibacillary) respiratory samples. ${ }^{10,11}$

It is not prone to cross-contamination, requires minimal biosafety facilities, can be performed by technicians with little training;

However, a recent meta-analysis reported the pooled sensitivity and specificity of GeneXpert in TPE as $46.4 \%$ and $99.1 \%$, respectively, compared with those of pleural fluid mycobacterial

Culture. ${ }^{12}$ However, there are limited data about the Xpert MTB/RIF assay using pleural fluid.

The most widely used diagnostic marker for TPE is the pleural fluid adenosine deaminase (ADA) level. ADA testing also gives same day result. It is a relatively easy, inexpensive and rapid, with pooled sensitivity and specificity estimates of $92 \%$ and $90 \%$, respectively, across different prevalence settings depending on the cut-point used. ${ }^{13}$ Since it is biomarkers of the inflammatory process in the pleural space and thereby do not confirm the etiologic agent.

So combinations of tests seem to perform better than any single test, especially combinations that include adenosine deaminase, Gene Xpert, total leukocytes count and lymphocytes in pleural fluid.

Therefore, the present study was done to evaluate and compare the role of Adenosine deaminase, Gene Xpert, total leukocytes count and lymphocytes in diagnosing tuberculous pleural effusion.

\section{Material and Methods}

The study population consisted of 100 suspected patients of tuberculous pleural effusion admitted in Chest \& TB ward of Government Medical College, KOTA from July 2019 to December 2019.

Pleural fluid samples were collected for routine microbiology, biochemical and cytological analysis. The samples were processed as follow:

Adenosine deaminase activity in pleural fluid was determined by colorimetric technique using the user defined method on a Roche Cobas Integra (Roche Diagnostics Ltd, Switzerland). Pleural fluid ADA levels greater than $30 \mathrm{U} / \mathrm{L}$, were reported as suggestive of pleural TB. ${ }^{14,15}$

Gene/xpert- $1 \mathrm{ml}$ aliquot of raw pleural fluid and a $1 \mathrm{ml}$ aliquot of concentrated pleural fluid (Prepared by centrifugation of $10-20 \mathrm{ml}$ pleural fluid at $3000 \times \mathrm{g}$ for 15 min, with the supernatant discarded and the pellet made up to $1 \mathrm{ml}$ with phosphate buffer solution) from each patient was diluted with $2 \mathrm{ml}$ of the Xpert MTB/RIF sample buffer. It was mixed vigorously and incubated at room temperature for $15 \mathrm{~min}$, finally run on the GeneXpert machine

For Cytology the slides were made from centrifuged deposits and stained with Giemsa. The total count was done by using modified neaubaur chamber and differential count was done on giemsa stained slides. Acid fast bacilli staining results were also documented

All patients were started on anti-tubercular therapy. They were followed up till the completion of treatment. Response to treatment was assessed on the basis of Chest X-ray and clinical findings.

\subsection{Inclusion criteria}

1. Patients of all age and sex with suspected tubercular pleural effusion (i.e. sign and symptoms -an acute febrile illness characterised by cough and pleuritic chest pain, night sweats, chills, weakness, dyspnoea, haemoptysis and weight loss)

2. Chest $\mathrm{X}$ ray findings of pleural effusion.

\subsection{Exclusion criteria}

Patients on anti-tubercular therapy

Diagnosed case of carcinoma of any site.

Transudative pleural effusion

\section{Objectives}

1. To determine the role of genexpert in the diagnosis of tubercular pleural effusion.

2. To study the association between pleural fluid CBNAAT, ADA and lymphocytes percentage.

\section{Results}

There were 65 male and 35 female patients, with a mean age of 40 years. Cough and low grade fever was the most common symptoms followed by weight loss and loss of appetite. CBNAAT was positive in 30 patients. ADA was 
increased ( $>30 \mathrm{IU})$ in 85 patients with a mean value of 65IU. 15 patients had value less than 30 . The lymphocytic predominance was found in 95 patients whereas in 5 patients neutrophils predominance was seen. The mean value for total leukocytes count was $720 \mathrm{cell} / \mu \mathrm{L}$. It was found that 12 patients with low ADA value has increased total leukocytes count and lymphocytic predominant. All the CBNAAT positive patients had increased ADA value and $100 \%$ lymphocytes, increased total leukocytes count. AFB staining was negative in all the cases. The culture was done in a few cases ${ }^{16}$ and was negative.

In the current study, increased ADA and lymphocytes predominance was seen in most of the patients. CBNAAT was less sensitive due to paucibacillary nature of the disease process.

\section{Discussion}

TB remains one of the most frequent causes of pleural effusions in developing countries like India. The gold standard for the diagnosis of tuberculous pleuritis remains the detection of M. tuberculosis in pleural fluid or pleural biopsy specimens, either by microscopy and/or culture, or the histological demonstration of caseating granulomas in the pleura along with AFB.

The results of present study show that Gene Xpert MTB assay play significant role in routine tuberculous pleural effusion diagnosis. The result is available in same day with high specificity. But it cannot be used alone for the diagnosis of TPE, given its low sensitivity. So it cannot be used alone.

Globally the use of GeneXpert assay has resulted in an increase in the number of positive results by $16.5 \%$ and this increase has been more important for the extra-pulmonary specimens especially the body fluids. ${ }^{17}$ It is considered a breakthrough in the diagnosis of TB and EPTB. One of the major limitations of this technique is that it cannot distinguish between viable and non-viable microorganisms. Hence it should not be used to monitor patients on treatment.

In our study, pleural fluid CBNAAT was found to be positive in only $30 \%$ of cases.

In a well-structured meta-analysis of 24 studies from India, it was seen that the sensitivity of CBNAAT in TPE was between $22.7-51.4 \%$ using a composite reference standard (CRS) and pleural fluid culture as the reference standard. ${ }^{18}$

In another meta-analysis, determining the role of genexpert in the diagnosis of EPTB, a total of 18 studies were analyzed with 4461 samples. Pooled sensitivity for pleural fluid was $46.4 \%$ against culture and $21.4 \%$ against CRS. ${ }^{19}$

Meldau et al. compared the diagnostic utility of ADA, genexpert and gamma interferon (IFN gamma). They proposed IFN to be as sensitive as ADA in high prevalence settings, and finally, concluded that either of the two could be used to guide therapy, as routine pleural biopsy may be challenging in high prevalent, resource-limited countries. ${ }^{20}$ Shukla et al found that sensitivity of genexpert in TPE was $20.58 \%$ in their study. Rifampicin resistance was detected in $21 \%$ of cases. They found a positive correlation with high ADA values, pleural fluid lymphocyte counts and MTB detection by genexpert. ${ }^{16}$

We conclude that a high adenosine deaminase (ADA) (> $40 \mathrm{U} / \mathrm{L}$ ) combined with Light's criteria to define exudates in a lymphocyte predominant effusion constitutes enough evidence to diagnose TPE, able to avoid pleural biopsy and initiate anti-tubercular therapy. ${ }^{21,22}$

That is, ADA $<40$ excluded tuberculosis in $90 \%$ of cases.

When interpreting ADA levels, the clinician must additionally be aware of situations which may increase the likelihood of both the false-negative and false-positive ADA results. In the early phase of the disease low levels of ADA in the pleural fluid may be found, giving rise to a false negative result.

Conversely, raised ADA levels may be observed in a number of conditions potentially leading to a false positive diagnosis of TB. These include rheumatoid effusion, empyema due to other bacteria, mesothelioma, lung cancer, parapneumonic effusion, and haematological malignancies. ${ }^{23,24}$

Also the diagnostic usefulness of ADA depends not only on its sensitivity and specificity, but also on the local prevalence of TB. In populations with a high prevalence of TB and clinical suspicion of TB effusion, elevated ADA level might be considered as a confirmatory test justifying treatment initiation. Specificity of ADA in low prevalence areas has also been estimated. In a study done in north-western Europe, 338 patients with effusions were analysed, and when an ADA cut-off of $35 \mathrm{U} / \mathrm{L}$ was used in combination with lymphocytic effusions, sensitivity was $90.9 \%$ and specificity was $98.9 \%{ }^{25}$

Thus it would be possible to establish the diagnosis of TPE with use of gene xpert, and ADA without the need for a pleural biopsy. Pleural biopsy should be reserved for patients with a low pleural fluid ADA, negative cytology and a high suspicion of a neoplasm or those suspected to have multiple drug- resistant TB. ${ }^{26}$

When a lymphocyte neutrophil ratio of 0.75 or greater is used in combination with ADA, the sensitivity, specificity, positive predictive value, negative predictive value, and efficiency for the identification of TB were reported at $88 \%, 95 \%, 95 \%, 88 \%$, and $92 \%$, respectively. ${ }^{27}$ CBNAAT is very specific as positive test indicate tuberculous lesion but negative result does not rule out the same. Out of 100 patients 95 patients responded to treatment. After 6months chest X-ray and clinical condition were evaluated. The response was not satisfactory among 5 patients. It may be due to irregular treatment or might have developed drug resistant. 


\section{Conclusion}

In India lymphocyte predominant effusions with high ADA levels (> $40 \mathrm{U} / \mathrm{L}$ ) and clinical suspicious of TB, where alternate diagnosis seems unlikely are treated as TPE in India.

Pleural biopsy being invasive is not feasible at all health care centres, it is not put into routine clinical practice.

Sensitivity of ADA when combined with lymphocyte predominant exudates, in high prevalence areas has stood valuable test in deciding the initiation of ATT. Low sensitivity of pleural fluid CBNAAT as shown in our study limits its clinical usefulness. But given its high specificity, it can potentially obviate the need for an invasive procedure in at least one fourth of patients with TPE.

The results of our study demonstrate that in regions with a high prevalence of tuberculosis, it is possible to establish the diagnosis of TPE from clinical data and pleural fluid analysis, with good diagnostic accuracy.

\section{Source of Funding}

None.

\section{Conflict of Interest}

None.

\section{References}

1. Light RW. Update on tuberculous pleural effusion. Respirol. 2010;15(3):451-8.

2. Seibert AF, Haynes J, Middleton R, Bass JB. Tuberculous Pleural Effusion. Chest. 1991;99(4):883-6.

3. Light RW. Useful tests on the pleural fluid in the management of patients with pleural effusions. Curr Opin Pulm Med. 1999;5(4):2459 .

4. Bueno CE. Cytologic and bacteriologic analysis of fluid and pleural biopsy specimens with Cope's needle. Study of 414 patients. Arch Internal Med. 1990;150(6):1190-4.

5. Seibert AF, Haynes J, Middleton R, Bass JB. Tuberculous Pleural Effusion. Chest. 1991;99(4):883-6.

6. Rahman NM, Ali NJ, Brown G, Chapman SJ, Davies RJ, Downer NJ, et al. Local anaesthetic thoracoscopy: British Thoracic Society Pleural Disease Guideline 2010. 2010;65(2:ii):54-60.

7. Dhooria S, Singh N, Aggarwal AN, Gupta D, Agarwal R. A Randomized Trial Comparing the Diagnostic Yield of Rigid and Semirigid Thoracoscopy in Undiagnosed Pleural Effusions. Respir Care. 2014;59(5):756-64.

8. Maturu VN, Dhooria S, Bal A, Singh N, Aggarwal AN, Gupta D, et al. Role of Medical Thoracoscopy and Closed-Blind Pleural Biopsy in Undiagnosed Exudative Pleural Effusions. J Bronchology Interv Pulmonol. 2015;22(2):121-9.

9. Diacon AH, de Wal BWV, Wyser C, Smedema JP, Bezuidenhout J, Bolliger CT, et al. Diagnostic tools in tuberculous pleurisy: a direct comparative study: 1. Eur Respir J. 2003;22(4):589-91.

10. Chang K, Lu W, Wang J. Rapid and effective diagnosis of tuberculosis and rifampicin resistance with Xpert MTB/RIF assay: a metaanalysis. J Infect. 2012;64(6):580-8.

11. Report of the Tenth Meeting WHO Strategic and Technical Advisory Group for Tuberculosis (STAG-TB); 2010
12. Alvarez-Uria G, Azcona JM, Midde M, Naik PK, Reddy S, Reddy R. Rapid diagnosis of pulmonary and extrapulmonary tuberculosis in HIV-infected patients. Comparison of LED fluorescent microscopy and the geneXpert MTB/RIF assay in a district hospital in India. Tubercs Res Treat. 2012;.

13. Liang QL, Shi HZ, Wang K, Qin SM, Qin XJ. Diagnostic accuracy of adenosine deaminase in tuberculous pleurisy: a meta-analysis. Respir Med . 2008;102(5):744-54.

14. Blake J, Berman P. The use of adenosine deaminase assays in the diagnosis of tuberculosis. S Afr Med J. 1982;62(1):19-21.

15. Soo Y, Singhal AB, Leung T, Yu S, Mak H, Hao Q, et al. Reversible Cerebral Vasoconstriction Syndrome with Posterior Leucoencephalopathy after Oral Contraceptive Pills. Cephalalgia. 2010;30(1):42-45. Available from: https://dx.doi.org/10.1111/j.14682982.2009.01868.x. do1:10.111//.1468-2982.2009.01868.x.

16. Shukla A, Kajal N, Malhotra B. Role of gene Xpert MTB/ RIF assay in diagnosis of Tubercular Pleural Effusion. Int J Curr Res Med Sci. 2017;3(5):105-10.

17. Alvarez-Uria G, Azcona JM, Midde M, Naik PK, Reddy S, Reddy R, et al. Rapid diagnosis of pulmonary and extrapulmonary tuberculosis in HIV-infected patients. Comparison of LED fluorescent microscopy and the geneXpert MTB/RIF assay in a district hospital in India. Tuberc Res Treat. 2012;

18. Sehgal IS, Dhooria S, Aggarwal AN. Diagnostic performance of xpert MTB/RIF in tuberculous pleural effusion: systematic review and metaanalysis. J Clin Microbiol. 2016;54(4).

19. Rosso F, Michelon CT, Sperhacke RD, Verza M, Olival L, Conde $\mathrm{MB}$, et al. Evaluation of real-time PCR of patient pleural effusion for diagnosis of tuberculosis. BMC Res Notes. 2011;4(1):279.

20. Meldau R, Peter J, Theron G. Comparison of same day diagnostic tools including Gene Xpert and unstimulated IFN-g for the evaluation of pleural tuberculosis: a prospective cohort study. BMC Pulm Med. 2014;

21. Goto M, Noguchi Y, Koyama H, Hira K, Shimbo T, Fukui T, et al. Diagnostic value of adenosine deaminase in tuberculous pleural effusion: a meta-analysis. Ann Clin Biochem. 2003;40(4):374-81.

22. Villena-Garrido V, Ferrer-Sancho J, ndez Blasco LH. Diagno'stico y tratamiento del derrame pleural (normative SEPAR). Arch Bronconeumol. 2006;42:349-72.

23. Valdés L, Pose A, and ESJ. Tuberculous pleural effusions. Eur J Intern Med. 2003;14:77-88.

24. Lee SJ, Kim HS, Lee SH. Factors influencing pleural adenosine deaminase level in patients with tuberculous pleurisy. Am J Med Sci. 2014;348:362-5.

25. Arnold DT, Bhatnagar R, Fairbanks LD, Zahan-Evans N, Clive AO, Morley AJ, et al. Pleural Fluid Adenosine Deaminase (Pfada) in the Diagnosis of Tuberculous Effusions in a Low Incidence Population. PLOS ONE. 2015;10(2):e0113047.

26. Valdes L, Alvarez D, Jose ES, Juanatey JR, Pose A, Valle JM, et al. Value of adenosine deaminase in the diagnosis of tuberculous pleural effusions in young patients in a region of high prevalence of tuberculosis. Thorax. 1995;50(6):600-3.

27. Burgess LJ, Maritz FJ, Roux IL, Taljaard JJF. Combined Use of Pleural Adenosine Deaminase With Lymphocyte/Neutrophil Ratio. Chest. 1996;109(2):414-9.

\section{Author biography}

Archana Garg Assistant Professor

Lakshmi Agarwal Assistant Professor

Mamta Gupta Assistant Professor

Rishab Mathur Student 
Cite this article: Garg A, Agarwal L, Gupta M, Mathur R. A

Prospective Study for comparison of diagnostic utility of Gene

XPERT MTB/RIF Assay, adenosine deaminase and cytology in

Tuberculous Pleural Effusion. IP Arch Cytol Histopathology Res 2020;5(3):194-198. 